\title{
Human Herpesvirus 5
}

National Cancer Institute

\section{Source}

National Cancer Institute. Human Herpesvirus 5. NCI Thesaurus. Code C112314.

A species of enveloped and spherical viruses with a capsid with $T=16$ icosahedral symmetry and surrounded by amorphous tegument, in the Herpesviridae family and Cytomegalovirus genus. The genome is composed of monopartite, linear doublestranded DNA. Human herpesvirus 5 is found in humans and causes mononucleosis-like symptoms in healthy individuals and pneumonia, hepatitis, encephalitis, myelitis, colitis, retinitis, neuropathy, mucoepidermoid carcinoma as well as other malignancies in immunocompromised persons, organ transplant recipients and newborn infants. Human herpesvirus 5 is transmitted cong enitally or via direct person-to-person contact. 